\title{
Analysis and presenting the educational techniques in Machine and Deep Learning Short communication
}

\author{
S Rahul ${ }^{1 *}$ \\ ${ }^{1}$ Research Scholar at KL Deemed to be University, Vaddeswaram, Green Fields, Guntur, India \\ *Corresponding author E-mail: 15304028@kluniversity.in
}

\begin{abstract}
This paper gives a present of general learning of deep methodology and its applications to a variety of signal and data proce ssing schedules. It is discussed about Machine learning vs. Deep Learning a brief and which is best suited in the market, Dissimilarities, Problem handling, Interpretability, Comparative and different options between cubic centimeter and metric capacity unit and concluded by justifying deep learning is a part of Machine learning and Machine learning is a part of Artificial intelligence.
\end{abstract}

Keywords: Artificial Intelligence; Cubic centimeter; Deep learning; Machine Learning; Metric Capacity.

\section{Introduction}

Since 2006, learning of deep structured, or more commonly called learning of deep or learning of hierarchical, has emerged as a new field of learning of machine research. The Discussion on Learning of machine compared to Learning of deep obtained considerable stream within some past duration. A basic strength of each this technology under lay in capacity so that a find out through-out there knowledge. Although each of those affects technologies in AI excel in "Learning algorithms," specific way within the Learning of Machine's step by step processes understanding is incredibly totally differ with the source that the educational ways in the Learning of Deep(DL) step by step process. milliliter instantly monitors knowledge themes which provides relations cooperatively, metric capacity unit step by step process of learning increasingly through involved flavors in information. Deciliter, taken into account, set metric capacity unit, wherever thought process initiates from a superimposed step by step process with interconnection of systems normally referred to a synthetic Netwo of Neural. Network of Artificial Neural nearest in compared with the brain of human in function of functioning. 2. Leaning of Machine compared to learning of deep: that in demand, clean approach of visualizing including intellectual rights within area in Learning of Machine compared to Learning of deep, nowadays can still prolong high in coming duration. By coming to Learning of Machine may be an explicit sort in Intelligence of Artificial that provides learning automatic of step by step processes through learning offered knowledge by self. Learning of Machine step by step processes, square measure good set of instructions which may go in, survey, also transform knowledge from knowledge with none intervention of programmatic. cc displays square measure usually utilized in trade sectors to predict risks and opportunities. It claims that massive knowledge wouldn't have happened if milliliter wasn't nearby to grasp price through it. In the opposite side, promotion of Gartner report revolution to rising advancements called as Learning of Deep together group of eight rising advancements of the year 2017, On the far side. Deep Learning is also thought of a set of metric capacity unit, wherever knowledge sinking occurs from bedded top down approach data disclosed. Article of the DATAVERSITY, the edges of the business, i.e., of learning of deep Membrane (similar in terms of the operation of the brain of human) Learning quality generally captured in metric capacity unit being represented all right.

\section{Approaches of learning:}

It may be that metric capacity unit step by step process of learning's affect guided knowledge whereas deciliter step by step process of learning's, square measure implemented on unattended paths. Following forecasts provided by Gartner: In 2019 , ten \% service of customer workers are involved into larva bi-visuals in the year of 2019, initiates can lead the economy of AI, departure giants behind the four. In coming 2020, bright of business can be upon psychological feature advancements. Although by 2020 , a minimum of twenty companies can interact workers among networks of neural. Co linearity amount Learning of Machine and Learning of Deep, Zen desk journal present a easy thanks for perceive Learning of Deep compared to Learning of Machine utilizes the content "data" combined milliliter, Metric unit of capacity. Consistent with this post, whereas metric capacity unit step by step process of learning through knowledge, so that form choices, either assumptions, deciliter step by step process of learning act along knowledge, flavors in form educational development, accumulative. A mutual data of each milliliter, metric capacity unit affect "learning algorithms," tho' the educational strategies square measure terribly totally different. The "common thread" between milliliter and metric capacity unit is made understand from a mutuality of a driver self automobile. Essential indications implies once we unable to create set of instructions for unravel issues, Knowledge's of intelligence of machine of milliliter otherwise metric capacity unit shifts to show systems turning to machines of self-solving. Other equality in milliliter , metric capacity unit underlay within applied areas in similar to laptop view, recognition of picture, data systems of retrieval, selling automatic, medicine of medical, wherever each 
milliliter, deciliter step by step processes of ought to be objectively clarified in an exceedingly Court of Law.

\section{Non nonlinearities in Learning of Machine and Learning of Deep:}

Specified before, least refinement amongst milliliter, deciliter inculcates in way of deal with growing for every situation. Examination Magazine of India shows "iterative learning process" used for milliliter varies start from superimposed way of learning captured in deciliter. Learning of deep Associate in Nursing Learning of Machine differences: Views of recent in a present conversation examines anyway metric limit unit models are outline to be completely "unsupervised" understudies - learning without anyone else - one layer at any given moment. As per Forbes the essential refinement between Machine Learning versus Profound Learning is inside the genuine way to deal with learning. Metric limit unit needs horrendously high volumes of data that calculations use to shape decisions concerning distinctive learning. Also, metric limit unit calculations are regularly connected to any sorts of learning - picture, sound, video, discourse, and so on, that isn't some of the time feasible with metric limit unit. Information Science Central takes a gander at the varieties between very surprising branches of data Science or AI somewhat extra comprehensively and this can be wherever per users can find the shifted operational ways to deal with taking care of business learning. A portion that of higher inequalities in comparison of Learning of Machine versus Profound educating are: dealing with: Metric limit unit, curiously big drawback divided in littler lumps, every piece explained separately. At long last, all arrangements square measure supplant along. In DL, the issue is illuminated complete to wrap up. Volume of data Usage: ML calculations have demonstrated their nerve each in huge information and extensive information projections. Profound Learning calculations will perform once the data volume is colossal. Anyway will any of those calculations extremely work once the data volume is low? Processor Requirements: ML calculations oversee great in ordinary machines anyway metric limit unit calculations need high-performing machines to perform well. Highlight Engineering: In metric limit unit, "include extraction" stays took care of physically, while in metric limit unit, include extraction happens mechanically all through the instructive technique. Since the manual technique is each time and work concentrated, metric limit unit has decreased a lot of work via computerizing this basic piece of highlight extraction. Preparing Time: In metric limit unit, due to included layers of neural, educating time is higher, extra progressed. Machine Learning, calculations is prepared to be told in an meet of an every an exceedingly in a terribly brief time.

\subsection{Interpretability:}

In milliliter, exact guidelines square measure offered by calculations to clarify choices the selections $\}$ behind particular decisions, while in deciliter, the decisions appear to be "discretionary," giving the client next to no informational ability to legitimize choices.

\section{Comparative and different options between cubic centimeter and metric capacity unit:}

Gartner claims that metric capacity unit algorithms square measure over determining beings in several themes just as recommendations of product, prediction of demand, works of speech-to-text, etc. Matured cases, metric capacity unit, metric capacity unit conjointly give the most effective outputs. Best applications level, metric capacity unit, supervised metric capacity unit step by step processes of learning produce equally, to illustrate each metric capacity unit and supervised metric capacity unit algorithms are often trained to spot teams of objects among pictures in a very vast image library. wherever metric capacity unit stands aside from metric capacity unit is that metric capacity unit will directly handle all kinds of information similar to Voice, images of liquidity, picture, But cubic centimeter got method of information, include info man of science to coach the algorithmic rule before creating trained modeled. The teaching-self capacity of metric capacity unit step by step processes of learning does terribly strong full. Scope retrieval method of cc remains maintained by an individual's information mortal that keeps an extra stress on the interval, efficiency. Automated retrieval of science feature obtainable, deciliter keeps it a couple of lightweight duration earlier than ancient metric capacity unit. Extreme duration, burden of labor obligatory on beings information professionals is additional excavated in metric capacity unit because the algorithms will mechanically specialize in right options with none intervention from somebody's soul. Relief done by feature automatic retrieval in metric capacity unit replied by the hierarchy style demand. Several deals, data professional are gains giant. Information bunches at the same time that keeps significant pressure on the output duration, potency. Reasonwhy, deciliter step by step processes of learning's best run in major computers capacity of process giant operations of vectors terribly fast. Versus to Learning of Machine, Big disadvantage in Deep Learning that needs terribly costly devices to execute. Which create Learning of Deep "affordable," to do business, information soul group developed views of graphic data process blocks (General Purpose Units), Might do millions of functions in no time. Other connected feature found within deciliter zone that member, ASCII text file data(s), that square measure canned answers for several picture, and audio identifying applies. Most important ASCII text file libraries on the market nowadays area unit Flow of tensor, MXNet or Cafee.

Extreme duration, burden of labor obligatory on beings information professionals is additional excavated in metric capacity unit because the algorithms will mechanically specialize in right options with none intervention from somebody's soul.

\section{Conclusion:}

The written of learning of deep Updates: Learning of Machines, Reinforcement of Deep Learning, and discusses boundaries however most recent developments within the areas of Intelligence of Artificial, metric capacity unit square measure step by step machines turning as a thinking self entries as beings.

If higher advancement assumption of Gartner's for this period of ten "all things digital" will convert to a implementable reality, Learning of Machine and Learning of Deep, within different good knowledgeableness, should-still higher, in potency, businesses worth, also functional stages.

\section{Acknowledgement}

I want to thank Bharat Institute of Engineering and technology for the support and time provided.

\section{References}

[1] "Machine Learning for Image Reconstruction" Ge Wang, Jong Chu Ye, Klaus Mueller, Jeffrey A. Fessler May 2, 2018.

[2] Temporal Dynamics of Learning for Resolution of super video: A Learning of deep Approach Ding Liu, Student Member, IEEE, Zhaowen Wang, Member, IEEE, Yuchen Fan, Xianming Liu, Zhangyang Wang, Member, IEEE, Shiyu Chang, Xinchao Wang, and Thomas S. Huang, Life Fellow, IEEE.

[3] Intelligent tuninf of parameter in Optimization-based Iterative CT Reconstruction via Deep Reinforcement Learning Chenyang Shen, Yesenia Gonzalez, Liyuan Chen, Steve B. Jiang, Xun Jia.

[4] Deep Learning: Applications and Methods Li Deng, Microsoft Research. One Microsoft Way Redmond, WA 98052, USA 
deng@microsoft.com, Dong Yu, Microsoft Research, One Microsoft way Redmond, WA 98052, USA, Dong.Yu@microsoft.com

[5] Deep Monocular Depth Estimation via Integration of Global and Local Predictions Youngjung Kim, Student Member, IEEE, Hyungjoo Jung, Student Member, IEEE, Dongbo Min, Senior Member, IEEE, and Kwanghoon Sohn, Senior Member, IEEE.

[6] Image Reconstruction Is a New Frontier of Machine Learning - Editorial for the Special Issue 